\title{
Effect of Alternaria solani Exudates on Resistant and Susceptible Potato Cultivars from Two Different pathogen isolates
}

\author{
Hadis Shahbazi ${ }^{1 *}$, Heshmatollah Aminian ${ }^{2}$, Navazollah Sahebani ${ }^{2}$ and Dennis Halterman ${ }^{3}$ \\ ${ }^{\prime}$ Young Researchers Club, Arak Branch, Islamic Azad University, PO Box 38135/567, Arak, Iran \\ ${ }^{2}$ Department of Plant Protection, Aboryhan Campus, Tehran University, P.O. Box 33955-159, Tehran, Iran \\ ${ }^{3}$ U.S. Department of Agriculture-Agricultural Research Service, Vegetable Crops Research Unit, Madison, WI 53706, USA \\ (Received on December 10, 2010; Accepted on February 12, 2011)
}

Early blight of potato, caused by Alternaria solani, is a ubiquitous disease in many countries around the world. Our previous screening of several Iranian potato cultivars found that significant variation in resistance phenotypes exists between two cultivars: resistant 'Diamond' and susceptible 'Granula'. Our previous analysis of five different pathogen isolates also identified varying degrees of aggressiveness regardless of the host cultivar. Here, a bioassay was used to study the role of liquid culture exudates produced in vitro on pathogenicity and elicitation of disease symptomology in seedlings as well as detached leaves. Responses of host genotypes to the exudates of the two $A$. solani isolates were significantly different. Detached leaves of the resistant cultivar 'Diamond' elicited fewer symptoms to each isolate when compared to the susceptible cultivar 'Granula'. Interestingly, the phytotoxicity effect of the culture filtrate from the more aggressive isolate $A$ was higher than from isolate $\mathbf{N}$ suggesting an increased concentration or strength of the toxins produced. Our results are significant because they indicate a correlation between symptoms elicited by $A$. solani phytotoxins and their aggressiveness on the host.

Keywords : Alternaria solani, bioassay, early blight, pathogenicity, phytotoxicity.

Early blight, caused by Alternaria solani is a serious and important plant disease in warm and humid regions (Scherf and MacNab, 1986). The main hosts of $A$. solani are solanaceous crops including tomato, potato, eggplant, and pepper (Ellis and Gibson, 1975; Neergaard, 1945). The disease is characterized by formation of dark brown lesions and necrosis of leaf tissue between the veins. Host colonization is facilitated by enzymes (cellulases and pectin methyl galacturonase) that degrade the host cell wall and by

\footnotetext{
*Corresponding author.

Phone) +00989183612921, FAX) +00988612238785

E-mail)sah.shahbazi@yahoo.com
}

phytotoxins that facilitate the necrotrophic growth habit of the fungus (Rotem, 1994). Fungi of the genus Alternaria produce host-specific and host non-specific toxins involved in virulence or pathogenesis, which affect structural components of the host tissues including the plasma membrane, chloroplasts, and mitochondria (Hanneke et al., 1988; Lynch et al., 1991; Matern et al., 1978; Otani et al., 1995). Eleven toxins have been identified in culture filtrates of A. solani (Montemurro and Visconti, 1992). Among these, alternaric acid and solanapyrone $\mathrm{A}, \mathrm{B}$, and $\mathrm{C}$ are able to induce necrotic symptoms similar to $\mathrm{EB}$ symptoms (Montemurro and Visconti, 1992). Alternaric acid is one of the major metabolites in the filtrates (Brian et al., 1952) and is probably the main metabolite responsible for the development of necrotic and chlorotic symptoms (Pound and Stahmann, 1951). This toxin, isolated from lesions or from culture filtrate, causes chlorosis and necrosis when introduced in tomato plants and also damages nonhosts of Alternaria such as cabbage, radish, spinach, pea, bean, and others, demonstrating its nonspecificity (Pound and Stahmann, 1951). It was concluded that alternaric acid alters the morphological and physiological characteristics of plasma membranes near plasmodesmata and thereby causes a permeability change, which leads to a leakage of electrolytes (Langsdorf et al., 1991). In cases of less virulent strains or young and therefore resistant leaves, the germinating hyphae of $A$. cassiae and $A$. alternata tend to spread over the intact leaf surface and the only sites of infection are dead cells, suggesting that penetration is preconditioned by the secretion of toxins (van Dyke and Trigano, 1987; Von Ramm, 1962). Those toxins can potentially be used to select for resistant genotypes in vitro, either in a purified form or as culture filtrates. The main condition for this is that the plant reaction to the components of the culture filtrate used is similar to the reaction towards $A$. solani.

The objective of this project is to better understand the pathogenic differences between isolates of $A$. solani on potato cultivars. We have previously determined the re- 
sistance phenotypes of nine potato cultivars to five isolates of Alternaria solani (Shahbazi et al., 2010). Two potato cultivars, resistant 'Diamond' and susceptible 'Granula', and two pathogen isolates with significantly different pathogenicity phenotypes, named $\mathrm{A}$ and $\mathrm{N}$, were selected for further analysis. Here, we monitored the effect of exudates from liquid grown cultures of $A$. solani isolates $\mathrm{A}$ and $\mathrm{N}$ on cultivars 'Diamond' and 'Granula' using whole seedlings and detached leaflets. We hypothesized that phytotoxins present in the liquid exudates of the two isolates would elicit distinct effects on the host plants. Our results indicate that isolates of $A$. solani exhibiting different degrees of pathogenicity produce phytotoxins with corresponding effects on potato.

\section{Materials and Methods}

Cultural conditions. Potato (Solanum tuberosum) seeds were planted in plastic pots $(30 \mathrm{~cm}$ diameter) containing soil and perlite under greenhouse conditions $\left(18-25^{\circ} \mathrm{C}\right)$ at Tehran University. Potato seed consisted of small whole tubers weighing approximately $50 \mathrm{~g}$ each. One seed piece was planted per pot. Tubers were treated with thiabendazole (tekto WP60\%, Golsam Gorgan Company, Tehran) before planting. Plants were irrigated once per day or as needed.

Alternaria solani isolation and spore production. Potato leaves infected with Alternaria solani were collected in 2008 from commercial potato fields in Iran. Two isolates of A. solani were recovered from sections of potato leaves with early-blight lesions. An isolate, named "A", was previously characterized as being highly aggressive on potato and a second isolate, named "N", was less aggressive (Shahbazi et al., 2010). The isolates were maintained on PDA at $20^{\circ} \mathrm{C}$ for 7 days. To induce sporulation, cultures were grown for 6 days at $23-25^{\circ} \mathrm{C}$ on PDA under nearultraviolet light (310-400 nm) with $16 \mathrm{~h} /$ day light. Conidia were collected by washing plates with sterile water and the resulting spore suspension was adjusted to a concentration of $10^{6}$ spores $/ \mathrm{mL}$.

Greenhouse test. Four plants (45 days old) of Solanum tuberosum cvs. 'Diamond' (resistant) and 'Granula' (susceptible) were inoculated separately by spraying with each isolate of $A$. solani at a concentration of $10^{6}$ spores $/ \mathrm{mL}$ or a sterile water control (Bokshi et al., 2003; Nadia et al., 2007). The plants were covered with clear plastic bags for $24 \mathrm{~h}$ to increase humidity and accelerate infection and then grown under normal conditions in the greenhouse at $26^{\circ} \mathrm{C}$ (Bokshi et al., 2003; Pelletier and Fry, 1989). The intensity of infection was recorded using the scale described in Table 1 (Rodríguez et al., 2007).
Table 1. Scale for evaluation of the foliar damage caused by Alternaria solani

\begin{tabular}{cl}
\hline \hline Rating & Description of Symptoms \\
\hline 10 & Spots on lower leaves \\
20 & Spots on most of the lower leaves \\
30 & Spots on all lower and some of the middle leaves \\
40 & Clearly developed blight lesions in lower leaves \\
50 & Blight lesions in lower leaves spread to some middle \\
& leaves \\
60 & Blight lesions developed in all inferior and most of the \\
70 & middle leaves \\
80 & Blight lesions developed in all lower and middle leaves \\
& and spread to upper leaves \\
100 & Total blight (death of the plant) \\
\hline
\end{tabular}

Culture filtrate. Small disks ( $2 \mathrm{~mm}$ diameter) from 1-week old PDA cultures were placed in $25 \mathrm{ml}$ of sterile liquid medium containing $1.0 \mathrm{~g}$ of $\mathrm{KH}_{2} \mathrm{SO}_{4}, 0.5 \mathrm{~g}$ of $\mathrm{MgSO}_{4}, 6.0 \mathrm{~g}$ of casein hydrolysate, $100 \mathrm{~g}$ of sucrose, $1 \mathrm{mg}$ of $\mathrm{FeSO}_{4}$, $0.15 \mathrm{mg}$ of $\mathrm{CuSO}_{4}, 0.10 \mathrm{mg}$ of $\mathrm{ZnSO}_{4}$, and $0.10 \mathrm{mg}$ of $\mathrm{Na}_{2} \mathrm{MoO}_{4}$ per liter of distilled water. The medium was adjusted to $\mathrm{pH} 4.9$ with $0.1 \mathrm{M} \mathrm{HCl}$ and autoclaved before inoculation. The $\mathrm{pH}$ remained at 4.9 after autoclaving. The cultures were incubated for 6 weeks at $24^{\circ} \mathrm{C}$ in the dark without shaking (Maiero et al., 1991). The contents of the flasks were filtered through the Whatman no. 4 filter paper. Culture filtrates were stored at $4{ }^{\circ} \mathrm{C}$ in the dark up to 10 hours until used.

Phytotoxicity tests of culture filtrates on potato leaves in vitro. Aqueous dilutions of $2: 1,5: 1,10: 1 \mathrm{v} / \mathrm{v}$ (culture filtrate: sterilized water) were prepared and autoclaved for $15 \mathrm{~min}$. In earlier studies of Alternaria toxic metabolites, autoclaving did not affect phytotoxicity of culture filtrates (Pound and Stahmann, 1951). Control treatments included dilutions of noninoculated growth media with distilled water. A method similar to that described by (Hernandez et al., 1991) was used; however, without removing the roots. Plantlets from each cultivar were inoculated by placing 45 day-old whole in vitro plantlets in a $15 \times 2 \mathrm{~cm}$ test tube containing $5 \mathrm{ml}$ of the culture filtrate dilutions or control treatments containing corresponding dilutions of noninoculated media. Leaflets of 4-week old grown plants were excised and placed in plastic petri dishes on 9-cm discs of filter paper saturated with $3 \mathrm{ml}$ of culture filtrate dilutions or control treatments containing corresponding dilutions of noninoculated media (Gilchrist and Grogan, 1976; Hanneke et al., 1988; Siler and Gilchrist, 1983). The petri dishes were sealed with parafilm. The test tubes and petri dishes were placed in a growth chamber for $72 \mathrm{~h}$ at $22 \pm 2^{\circ} \mathrm{C}$, with 
Table 2. Scale for evaluation of the damage produced by $A$. solani culture filtrate in potato in vitro plantlets (in tubes)

\begin{tabular}{cl}
\hline \hline Rating & Description of symptoms \\
\hline 0 & No visible symptoms \\
1 & Light chlorosis at basal zone of plantlet \\
2 & $\begin{array}{l}\text { Advanced chlorosis at basal zone of plantlet } \\
\text { Basal zone slightly necrotic and some leaves of the mid- } \\
\text { dle zone show light chlorosis }\end{array}$ \\
4 & $\begin{array}{l}\text { Accentuated necrosis at basal zone and advanced chlo- } \\
\text { rosis in the middle zone of plantlet }\end{array}$ \\
5 & $\begin{array}{l}\text { Dead leaves at basal zone, middle zone slightly necrotic } \\
\text { and apical zone with light chlorosis }\end{array}$ \\
6 & $\begin{array}{l}\text { Plantlet necrotic up to the middle zone and apical zone } \\
\text { with advanced chlorosis }\end{array}$ \\
7 & Total collapse of plantlet \\
\hline
\end{tabular}

a photosynthetic photon flow density of $100 \mathrm{lE} / \mathrm{m} / \mathrm{s}$ and a day length of $16 \mathrm{~h}$. To evaluate the individual response of in vitro plantlets to the culture filtrate in tubes, a scale of 0-7 degrees was used (Table 2; Rodríguez et al., 2007). The detached leaflets were rated for the degree of phytotoxicity in petri dishes on a scale of zero to three in which $0=$ no symptoms, $1=$ slight necrosis, $2=$ moderate necrosis and wilting, and $3=$ severe necrosis and wilting (Maiero et al., 1991).

Analysis of results. All experiments were carried out using a factorial model of a completely randomized experimental design in four replicates per treatment. In greenhouse evaluations, the factorial model was $2 \times 3$ (two potato cultivars $\times$ three treatments), in culture filtrate tests it was $2 \times 3 \times 3$ (two cultivars $\times$ three treatments $\times$ three dilutions). Analysis of variance was performed on data from whole plant inoculation experiments using SAS v9. A Kruskal-Wallis analysis of variance was performed on in vitro phytotoxicity tests due to the nonparametric data sets. All data are presented as means of at least four replications.

\section{Results}

Greenhouse evaluation. Greenhouse infection assays of two potato cultivars with different isolates of $A$. solani revealed significant differences between hosts. S. tuberosum cv. 'Diamond' exhibited lower disease severity with an average score ( \pm std. error) of $16( \pm 2)$ among the two $A$. solani isolates (Fig. 1). Cultivar 'Granula' showed higher disease severity with an average score of $51( \pm 2)$. Variance analysis indicated a significant difference between responses of these two cultivars to A. solani infection as well as a significant difference from the water-inoculated control plants, which exhibited no visible symptoms. Between two

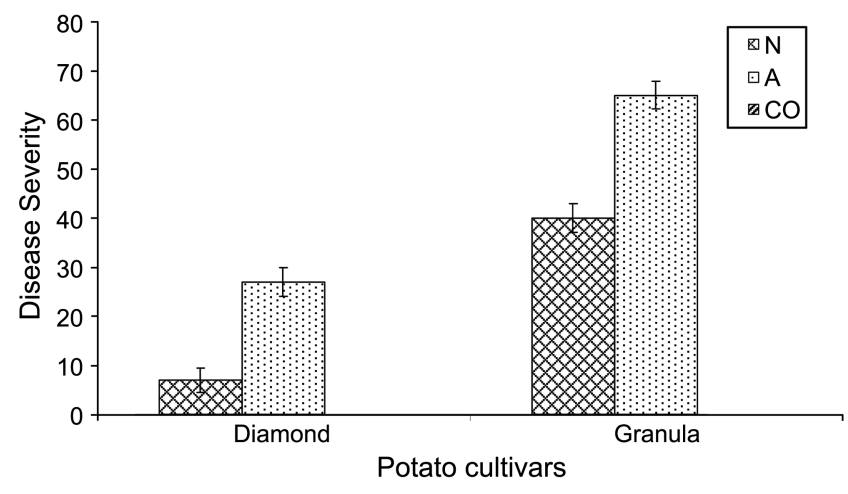

Fig. 1. Disease severity scores of greenhouse-grown potato cultivars inoculated with $A$. solani isolates ( $\mathrm{N}$ and $\mathrm{A}$ ) or water (CO). Error bars show the standard error among four replications.

A. solani isolates used in these assays, isolate A exhibited higher aggressiveness with an average host disease severity score of $45( \pm 2)$. Isolate $\mathrm{N}$ was less aggressive with an average host score of $24( \pm 2)$.

Culture filtrate in tubes. In order to assess whether EB disease symptoms in potato are caused by exposure to toxins exuded during fungal growth, we exposed whole plantlets to exudates of liquid-grown $A$. solani cultures. Chlorotic and necrotic symptoms developed in in vitro plantlets within $72 \mathrm{~h}$ after exposure. The severity of symptoms was higher in plantlets exposed to dilutions of the A. solani culture filtrate when compared to the control (Fig. 2). The affect on cv. 'Diamond' was lower than cv. 'Granula' with all dilutions (2:1, 5:1, and 10:1) of $A$. solani exudates. Differences in symptoms were observed between

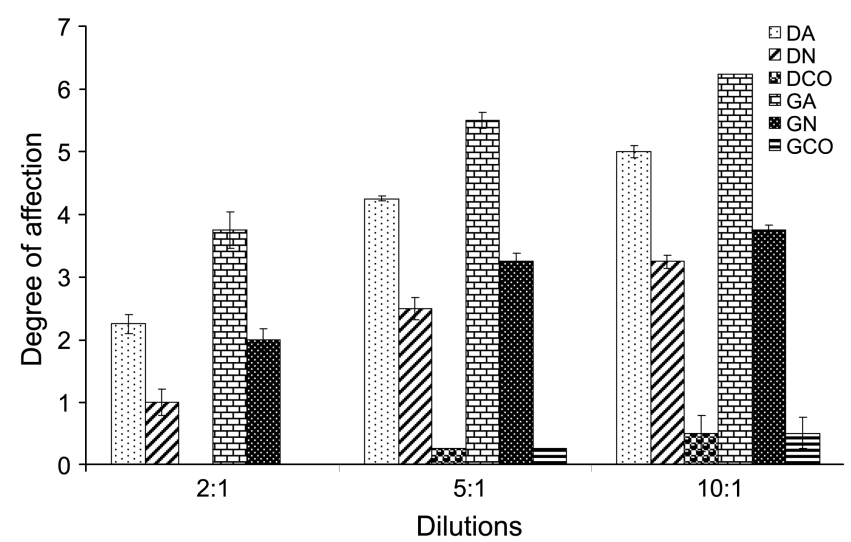

Fig. 2. Phytotoxicity tests of culture filtrates on plantlets in vitro. DCO: 'Diamond' in non-inoculated medium culture; GCO: 'Granula' in non-inoculated medium culture; GA: 'Granula' in culture filtrate of $A$. solani isolate A; DA: 'Diamond' in culture filtrate of $A$. solani isolate A; DN: 'Diamond' in culture filtrate of A. solani isolate $\mathrm{N}$; GN: 'Granula' in culture filtrate of $A$. solani isolate N. Results are the means of four replications. Error bars denote standard error. 


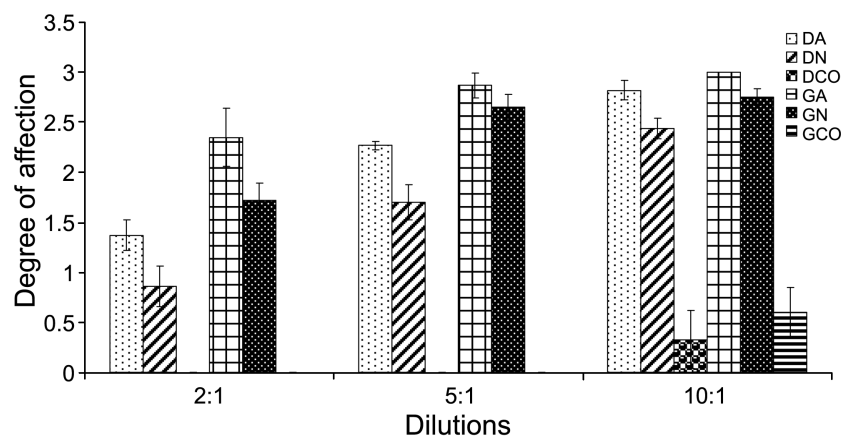

Fig. 3. Phytotoxicity tests of culture filtrates on potato leaves in petri plates. DCO: 'Diamond' in non-inoculated medium culture; GCO: 'Granula' in non-inoculated medium culture; GA: 'Granula' in culture filtrate of $A$. solani isolate A; DA: 'Diamond' in culture filtrate of $A$. solani isolate A; DN: 'Diamond' in culture filtrate of $A$. solani isolate N; GN: 'Granula' in culture filtrate of A. solani isolate N. Results are the means of four replications. Error bars denote standard error.

the two cultivars, but were not statistically significant. In both cultivars, symptom expression increased with a higher concentration of fungal culture filtrate and differences between dilutions were significant at $\mathrm{P}=0.017$.

Differences in host symptom expression were also observed when using isolates of $A$. solani with different aggressiveness phenotypes (Fig. 2). Culture exudate from A. solani isolate $\mathrm{A}$, which is more aggressive, elicited more symptoms on both cvs. 'Diamond' and 'Granula' than the less aggressive isolate $\mathrm{N}$ and this difference was significant at $\mathrm{P}<0.001$. The symptoms elicited by the two exudates were also significantly different from those elicited by the control regardless of the dilution used. In an analysis of reactions between isolates, dilution of the exudate of isolate $\mathrm{N}$ at a ratio of 5:1 (80\%) resulted in approximately the same symptoms as the exudate from isolate A diluted 2:1 (66.7\%). Compared to the fungus-free control, both culture filtrates of $A$. solani isolates elicited significantly more symptoms regardless of the dilution.

Culture filtrate in petri dishes. We also assessed the reaction of mature detached leaves to exposure to fungal exudates. In these experiments, the degree of symptoms in leaves of resistant cv. 'Diamond' was significantly less when compared to leaves of cv. 'Granula' $(\mathrm{P}=0.010)$. Differences in symptoms elicited by the two $A$. solani isolates were less pronounced in detached leaves compared to the wholeplant assays although the distinction was still significant at $\mathrm{P}<0.001$ (Fig. 3). Symptom expression was also higher than in the control-inoculated leaflets regardless of the isolate. Dilution of the fungal exudates also resulted in significant differences in symptoms in both 'Diamond' and 'Granula' leaflets with more symptoms observed in the most concentrated solution of fungal exudate $(\mathrm{P}<0.001)$.

\section{Discussion}

We have previously shown that there are significant differences among resistance phenotypes of potato cultivars 'Diamond' and 'Granula', as well as multiple cultivars with intermediate phenotypes (Shahbazi et al., 2010). These differences are likely not monogenic traits since a gradient of resistance phenotypes were observed when multiple cultivars were tested. We have also observed significant differences among isolates of $A$. solani. Two of these isolates, $\mathrm{A}$ and $\mathrm{N}$, produce significantly more and fewer, disease symptoms, respectively. The phenotypic differences in resistance correlate with an significant increase in phenolic compounds (Shahbazi et al., 2010) and expression of $\beta-1,3$ glucanase activity (Shahbazi et al., unpublished data), suggesting that these responses have a role in early blight resistance.

Here, we have shown that pathogenesis of $A$. solani in potato is also correlated with its ability to produce toxin metabolites that elicit disease symptoms in the absence of the pathogen. A. solani isolates with higher (A) and lower $(\mathrm{N})$ degrees of aggressiveness were significantly separated by disease severity scores as well as the symptoms elicited by their toxins in detached leaflet assays. Rodríguez et al. (2007) showed that culture medium of A. solani causes leaf and stem necrosis in in vitro plantlets of Solanum sp. that are phenotypically very similar to those caused by infection by spores. This phenomenon has also been observed elsewhere can be used effectively to select for individuals with increased resistance to early blight (Hernandez et al., 1991; Martinez and Sinclair, 1994; Veitía et al., 2001). Our results support the conclusion that different cultivars of potato contain variable reactions to $A$. solani toxins. Additionally, we have shown that different isolates of $A$. solani can produce toxins that produce more or fewer symptoms on the host, suggesting variability in the quantity or effectiveness of the toxins that are produced.

Two A. solani isolates with significantly different degrees of aggressiveness were identified using greenhouse infection assays. The $\mathrm{A}$ isolate induced more disease and $\mathrm{N}$ induced less disease, regardless of the host cultivar used. Although genotypic differences have not been established for these isolates, the presence of multiple strains of $A$. solani in potato growing regions is not uncommon (Lourenço et al., 2009; van der Waals et al., 2004). Isolates from the same host, such as potato, have been found to be more closely related to one another than those collected from another host, such as tomato (Weir et al., 1998). Therefore, among isolates collected from the same host, any genetic variability 
that translates to differences in aggressiveness is of great interest and warrants further study.

The two cultivars 'Diamond' and 'Granula' exhibited different levels of resistance to the two isolates using a detached leaflet assay. Together, differences in interaction between resistant 'Diamond' and susceptible 'Granula' with A. solani after 72 hours, suggest that their responses are closely associated with defense responses and that the differences in phenotypes are likely due to variability in sensitivity to metabolites produced during growth. The generation of phytotoxins has been demonstrated in several plant pathogens including individuals from the genera Aspergillus, Penicillium, Fusarium, Claviceps, and Verticillium (Svabova and Lebeda, 2005). To accelerate disease resistance evaluation, in vitro selection of toxin sensitivity has been successfully applied in several crops such as banana, carnation, grapevine, strawberry and wheat (Svabova and Lebeda, 2005). Here we have shown that an increased sensitivity to toxins produced by $A$. solani correlates with increased susceptibility to the pathogen. Furthermore, this sensitivity could be assayed using seedlings as young as 45 days old. Further work is required to determine whether the correlation between toxin sensitivity and resistance can be expanded to other potato cultivars or wild species of potato. We expect that the use of seedlings for in vitro evaluation of $A$. solani toxin sensitivity could provide a useful method for early generation selection of individuals in families segregating for resistance to early blight.

\section{Acknowledgements}

The authors are grateful to Dr. Etebarian (Department of Plant Protection, Aboryhan Campus, Tehran University, Tehran, Iran) for helpful suggestions. The authors also gratefully acknowledge Mr. Lak for his valuable support in the study.

\section{References}

Bokshi, A., Morris, S. and Deverall, B. 2003. Effects of benzothiadiazole and acetylsalicylic acid on $\beta$-1,3-glucanase activity and disease resistance in potato. Plant Pathol. 52:22-27.

Brian, P. W., Elson, G. W., Hemming, H. G. and Wright, J. M. 1952. The phytotoxic properties of alternaric acid in relation to the etiology of plant diseases caused by Alternaria solani (Ell. \& Mart.) Jones \& Grout. Ann. Appl. Biol. 39:308-321.

Ellis, M. G and Gibson, I. A. S. 1975. Alternaria solani. Commonwealth Mycological Institute, Kew, Surrey, UK.

Gilchrist, D. G. and Grogan, R. G. 1976. Production and nature of a host-specific toxin from Alternaria alternata f.sp. lycopersici. Phytopathology 66:165-171.

Hanneke, M. A., Witsenboer, C. E., Schaik, V., Bino, R. J., Löffler, H. J. and Jackes, H. 1988. Effects of Alternaria alternata f. sp. lycopersici toxins at different levels of tomato plant cell development. Plant Sci. 56:253-260.

Hernandez, M. M., Kowalski, B., Lorenzo, P. and Ortiz, U. 1991. Effectiveness of the filtrate use of Alternaria solani (Ellis and Martin) ( $\mathrm{J}$ and $\mathrm{G})$ in the selection in vitro of resistance forms. Cult. Trop. 12:48-50.

Langsdorf, G, Park, P. and Nishimura, S. 1991. Investigations on Alternaria solani infections: Effect of alternaric acid on the ultrastructure of tomato cells. Ann. Phyto. Soc. Jap. 57:32-41.

Lourenço, V., Moya, A., González-Candelas, F., Carbone, I., Maffia, L. A. and Mizubuti, E. S. G. 2009. Molecular diversity and evolutionary processes of Alternaria solani in Brazil inferred using genealogical and coalescent approaches. Phytopathology 99:765-774.

Lynch, D. R., Coleman, M. C. and Lyon, G. D. 1991. Effect of Alternaria solani culture filtrate on adventitious shoot regeneration in potato. Plant Cell Rep. 9:607-610.

Maiero, M., Bean, G A. and Ng, T. J. 1991. Toxin production by Alternaria solani and its related phytotoxicity to tomato breeding lines. Phytopathology 81:1030-1033.

Martinez, P. R. and Sinclair, M. 1994. Selection in vitro of resistance to early blight (Alternaria solani Sorauer.) in creole potatoes (Solanum phureja Junz). Fitopatol. Colomb. 18:90100.

Matern, U., Strobel, G and Shepard, J. 1978. Reaction to phytotoxins in a potato population derived from mesophyll protoplasts. Proc. Natl. Acad. Sci. USA. 75:4935-4939.

Montemurro, N. and Visconti, A., 1992. Alternaria metabolites chemical and biological data. In: Alternaria biology, plant disease and metabolites, ed. by J. Chelkowski and A. Visconti, pp. 449-558. Elsevier, Amsterdam, Netherland.

Nadia, G, El-Gamal, G., Abd-El-Kareem, F., Fotouh, Y. and ElMougy, N. 2007. Induction of systemic resistance in potato plants against late and early blight diseases using chemical inducers under greenhouse and field conditions. Res. J. Ag. Bio. Sci. 3:73-81.

Neergaard, P. 1945. Danish species of Alternaria and Stemphylium: taxonomy, parasitism, economical significance. Munksgaard, Copenhagen, Denmark, $560 \mathrm{pp}$.

Otani, H., Kohmoto, K. and Kodama, M. 1995. Alternaria toxins and their effects on host plants. Can. J. Bot. 73:453.

Pelletier, J. and Fry, W. 1989. Characterization of resistance to early blight in three potato cultivars: Incubation period, lesion expansion rate, and spore production. Phytopathology 9:511517.

Pound, G S. and Stahmann, M. A. 1951. The production of a toxic material by Alternaria solani and its relation to early blight disease of tomato. Phytopathology 41:1104-1114.

Rodríguez, N. V., Kowalski, B., Rodríguez, L. G, Caraballoso, I. B., Suárez, M. A., Pérez, P. O., Quintana, C. R., González, N. and Ramos, R. Q. 2007. In vitro and ex vitro selection of potato plantlets for resistance to early blight. J. Phytopath. 155:582-586.

Rotem, J. 1994. The genus Alternaria: biology, epidemiology, and pathogenicity. APS Press, St. Paul, Minnesota, USA, 326 pp.

Scherf, A. F. and MacNab, A. A. 1986. Vegetable diseases and 
their control. Wiley, New York, USA, 729 pp.

Shahbazi, H., Aminian, H., Sahebani, N. and Halterman, D. 2010. Biochemical evaluation of resistance responses of potato to different isolates of Alternaria solani. Phytopathology 100: 454-459.

Siler, D. J. and Gilchrist, D. G 1983. Properties of host specific toxins produced by Alternaria alternata f. sp. lycopersici in culture and in tomato plants. Phys. Plant Pathol. 23:265-274.

Svabova, L. and Lebeda, A. 2005. In vitro selection for improved plant resistance to toxin-producing pathogens. J. Phytopath. 153:52-64.

van der Waals, J. E., Korsten, L. and Slippers, B. 2004. Genetic diversity among Alternaria solani isolates from potatoes in South Africa. Plant Dis. 88:959-964.

van Dyke, C. G and Trigano, R. N. 1987. Light and scanning electron microscopy of the interaction of the biocontrol fungus Alternaria cassiae with sicklepod (Cassia obtusifolia). Can. J. Plant Pathol. 9:230-235.

Veitía, N., Dita, M. A., García, L., Herrera, L., Bermúdez, I., Acosta, M., Clavero, J., Orellana, P., Romero, C. and García, L. 2001. Use of tissue culture and the mutagenesis in vitro for the improvement of the resistance to Alternaria solani (Solanum tuberosum Lin.), to var Desirée. Biotechnol. Veg. 1:43-47.

Von Ramm, C. 1962. Histological studies of infection by Alternaria longipes on tobacco. Phytopath. Zeit. 45:391-398.

Weir, T. L., Huff, D. R., Christ, B. J. and Romaine, C. P. 1998. RAPD-PCR analysis of genetic variation among isolates of Alternaria solani and Alternaria alternata from potato and tomato. Mycologia 90:813-821. 